\title{
Systems Theory in an Analytic Setting
}

\author{
Manuela L. Bujorianu, Marius C. Bujorianu, and Howard Barringer
}

\begin{abstract}
Systems theory has almost a century of existence. The rapidly expanding research development in areas like hybrid and cyber physical systems has raised new concepts and techniques that have not been integrated in systems theory. Examples include concepts like simulation/bisimulation, hierarchical evolution and control, and so on. In their nature, these concepts are highly interdisciplinary and transdisciplinary making difficult their study in a uniform way.

In this paper, we propose a new doctrine for systems theory for capturing the essential properties of systems with complex dynamics like hybrid or stochastic behaviours. Our departing point is the basic idea from specification languages like VDM and $Z$, and modelling languages like UML and Modelica. The fundamental concept is that of symbolic dynamics where the state space can carry not only algebraic but also an analytic structure.
\end{abstract}

Keywords: symbolic dynamics, Markov model, simulation morphism, category theory.

\section{INTRODUCTION}

The purpose of systems theory is to make blurred the boundaries of disciplines by finding out the most important characteristics. With the rapid advances in computer science and control engineering in the context of hybrid and cyberphysical technologies, systems theory remains with a large palette of open problems. The first steps were made in frameworks like denotational semantics and category theory. These approaches deal only with deterministic systems, and they are not easy to be understood and extended for stochastic systems. The categorical approach from [10], [1] has been extended to stochastic hybrid systems in [8], but the development for stochastic systems was minor compared with the development in the deterministic case. This is mainly due to the completely different nature of the mathematics involved. Discovering the common principles of deterministic and stochastic behaviors of hybrid systems is a key point for developing modern systems theoretic concepts for stochastic hybrid systems [7].

Beyond universality, we are interested in a systems theory based on the mathematical principles underlying stochastic and nonlinear behaviors.. In the past decades, the stochastic control of nonlinear and hybrid systems [2] has been soundly founded on the theory of semigroups. This theory is now mature, rich and well respected. The problem with the semigroup approach is that it offers very little understanding

Manuela Bujorianu is with CICADA, School of Mathematics, University of Manchester, UK, E - mail : Manuela.Bujorianu@manchester.ac.uk

Marius Bujorianu is with CICADA, School of Mathematics, University of Manchester, UK, E - mail : Bujorianu@yahoo.com

Howard Barringer is with CICADA, School of Computer Science, University of Manchester, UK, E - mail : Howard.Barringer@manchester.ac.uk

This work has been funded by the CICADA project EP/E050441/1. in terms of systems, and it is completely disconnected from any theory of computation. It is a major challenge to make the theory of semigroups applicable to hybrid/cyberphysical systems or to other systems performing control and computation in continuous nonlinear environments. With this respect, the approach proposed in this paper is completely new and very challenging.

The approach we propose is centered on a new concept called symbolic dynamics. The dynamical systems modelled by this concept have a state space carrying algebraic and possibly analytical structures. This is common to the object orientation paradigm from software engineering, where data encapsulation gives rise to state models with algebraic structures. Specification languages like VDM and Z, and modelling languages like UML and Modelica offer formal tools for describing the state algebraic structures and to investigate their properties. For symbolic dynamical systems, it is less intuitive how system behaviors are captured over state spaces like spaces of functions or measures. In fact, these behaviors constitute symbolic representations for real life systems. The functions and measures have very versatile modelling power making the resulting concept suitable for systems axiomatization.

The symbolic dynamics have a clear intuition in terms of transition systems, unlike the semigroups of operators. Moreover, these dynamics can be defined on state spaces that can be finite, countable, continuous with multiple dimensions (like the Euclidean spaces), but most remarkably also on infinite dimensional spaces. Any realistic model of a physical system is based on the mathematics of infinite dimensional spaces [17], [11]. We develop the basic of a system theory around a concept of symbolic dynamics that is based on concepts like resolvent, Lyapunov functions, and a special form of semigroups called sweepings. The sweeping concept formalizes a remarkable procedure for solving partial differential equations introduced by Henri Poincare. In this way, the analytical tools of stochastic control are becoming available in the new theory.

This paper describes the fundamentals of an abstract theory for both discrete and continuous systems, deterministic or stochastic. The switching mechanism used in hybrid control systems requires a more elaborate construction, which is described in an accompanying paper [4]. In a follow-on paper, we will define composition operators for systems like product, feedback, concurrent composition and projective limits. The work presented here focuses on fundamental concepts like simulation, bisimulation, stochastic reachability and a new form of system equivalence.

The paper is structured as follows. The next section 
provides background material on Markov processes. In Section III, the symbolic dynamics are introduced and some classes are identified and investigated. In Section IV, we investigate the system theoretic properties of deterministic and stochastic continuous time systems. In Section V, we study the properties of bisimulation for the setting defined in the previous section. Bisimulation for continuous dynamics is in general hard to be understood. In general terms, continuous bisimulation is obtained from the definition of the discrete concept, by replacing "the discrete transitions" with "continuous trajectories". A further insight offered by the algebraic approach is related to the number of variables used to describe the system. This interpretation corresponds to the model order reduction from control engineering. For symbolic dynamics, the algebraic concept of bisimulation does not suffice. A state space model can be replaced by another one with a completely different analytical structure, while the dynamics remain equivalent. For example, one may consider a state space of functions with more integrability properties that can ease the study of reachability. Or, for the purpose of studying stochastic stability, one can replace a state space of functions by a space of measures. Such changes in representation can not be captured by the concept of bisimulation from computer science, although the dynamics can be the same. The paper ends with some final remarks.

\section{MARKOV PROCESSES}

Let us consider $\mathbf{M}=\left(x_{t}, P_{x}\right)$ a Markov process with the state space $X$. A Markov process retains no memory of where it has been in the past. Standard definitions can be find in any textbook like[5], [6]. Let $\mathcal{F}$ and $\mathcal{F}_{t}$ be the appropriate completion of $\sigma$-algebras $\mathcal{F}^{0}=\sigma\left\{x_{t} \mid t \geq 0\right\}$ and $\mathcal{F}_{t}^{0}=\sigma\left\{x_{s} \mid s \leq t\right\} . \mathcal{F}_{t}$ describes the history of the process up to the time $t$. Technically, with any state $x \in X$ we can associate a probability space $\left(\Omega, \mathcal{F}, P_{x}\right)$ where $P_{x}$ is such that its initial probability distribution is $P_{x}\left(x_{0}=x\right)=1$.

We adjoin an extra point $\Delta$ (thought as a cemetery/absorbing state) to $X$ as an isolated point, $X_{\Delta}=X \cup\{\Delta\}$. The existence of $\Delta$ is assumed in order to have a probabilistic interpretation of $P_{x}\left(x_{t} \in X\right)<1$, i.e. at some 'termination time' $\zeta(\omega)$ when the process $\mathbf{M}$ escapes to and is trapped at $\Delta . X$ is equipped with the Borel $\sigma$-algebra $\mathcal{B}(X)$ or shortly $\mathcal{B}$. Consider the set $\mathbf{B}(X)$ of bounded real measurable functions defined on $X$, which is a Banach space with the sup-norm $\|\varphi\|=\sup _{x \in X}|\varphi(x)|, \varphi \in \mathbf{B}(X)$. Suppose we have given a $\sigma$-finite measure $\mu$ on $(X, \mathcal{B})$.

Let $\mathcal{P}=\left(P_{t}\right)_{t>0}$ denote the semigroup of operators associated to $\mathbf{M}$, which maps $\mathbf{B}(X)$ into itself given by

$$
P_{t} f(x)=E_{x} f\left(x_{t}\right), \forall x \in X
$$

where $E_{x}$ is the expectation w.r.t. $P_{x}$.

Recall that a nonnegative function $f \in \mathbf{B}(X)$ is called $\alpha$ excessive $(\alpha \geq 0)$ if $e^{-\alpha t} P_{t} f \leq f$ for all $t \geq 0$ and $e^{-\alpha t} P_{t} f \nearrow f$ as $t \searrow 0$. If $\alpha=0$, a 0 -excessive function is simply called excessive function. Let us denote the cone of excessive functions by $\mathcal{E}_{\mathrm{M}}$. In the theory of Markov processes, the excessive functions play the role of the superharmonic functions from the theory of partial differential equations (for e.g. a function $f \geq 0$ is superharmonic w.r.t. the Laplace operator if $\Delta f \leq 0)$. Note, that the definition of excessive functions can be given in terms of the operator resolvent $\mathcal{U}$, which is the Laplace transform of $\mathcal{P}$.

The operator resolvent $\mathcal{V}=\left(V_{r}\right)_{r \geq 0}$ associated with $\mathcal{P}$ is

$$
V_{r} f(x)=\int_{0}^{\infty} e^{-r t} P_{t} f(x) d t, f \in \mathbf{B}(X), x \in X .
$$

When $r=0$, the operator $V_{0}$ is denoted by $V$, and is called the kernel operator.

Assumption 1: Suppose that $\mathbf{M}$ is a transient Markov process, i.e. there exists a strict positive Borel measurable function $q$ such that $V q$ is a bounded function.

The transience of $\mathbf{M}$ means that for any Borel set $E$ in $X$ and for almost all trajectories there exists a finite stopping time $t^{*}$ such that $x_{t} \notin E$ for all $t>t^{*}$. The transience hypothesis ensures that the excessive cone $\mathcal{E}_{\mathrm{M}}$ is not trivial. The infinitesimal generator $\mathcal{L}$ is the derivative of $P_{t}$ at $t=0$. Let $D(\mathcal{L}) \subset \mathbf{B}(X)$ be the set of functions $f$ for which the limit $\lim _{t \searrow 0} \frac{1}{t}\left(P_{t} f-f\right)$ exists (and it is denoted by $\mathcal{L} f$ ).

\section{SYMBOLIC DYNAMICS}

In this section, we define the fundamental concept of symbolic dynamics, and investigate examples and properties.

Definition 1: A symbolic dynamics is a structure $(T, S, \Phi)$, where: (i) $(T,+, o)$ is an arbitrary monoid, called time monoid; (ii) $S=(S, \mho)$ is the state space, eventually carrying a symbolic structure $\mho$; (iii) $\Phi: T \times S \rightarrow S$ is a map such that: $\Phi(0, s)=s ; \Phi\left(t^{\prime}, \Phi(t, s)\right)=\Phi\left(t^{\prime}+t, s\right)$; (iv) determinism: $\Phi(t, s)=\Phi\left(t, s^{\prime}\right), \forall t>0 \Rightarrow s=s^{\prime}$; (v) some compatibility axioms relates $\Phi$ to the symbolic structure of $S$.

The symbolic structure $\mho \checkmark$ can be an internal operation (e.g. addition), or an internal relation (e.g. order), or an external operation (e.g. multiplication with scalars), or an aggregate of such structures.

Example 1: Let $S$ be a linear space and $T=\mathbb{R}$. Define $\Phi(t, s)=e^{t} \cdot s$

A real time symbolic dynamics is a symbolic dynamics with $([0, \infty),+, 0)$ as time. This sort of dynamics are abbreviated as RTS dynamics and denoted as $(S, \Phi)$. An RTS dynamics $(S, \Phi)$ is called: (a) topological, if its state space $S$ carries a topological space structure, which makes continuous the map $\Phi$ on its second variable; (b) analytical, if its state space $S$ has a Banach space structure, which makes continuous the map $\Phi$ on its second variable, in the norm topology; (c) Hilbertean, if its state space $S$ has a Hilbert space structure, which makes continuous the map $\Phi$ in the inner product topology.

Definition 2: A symbolic dynamics $(S, \Phi)$ has deadlock is there is a point $\Delta$ such that $\Phi(t, x)=\Delta \Rightarrow \Phi(s, x)=$ $\Delta, \forall s \geq t$.

Definition 3: A latticeal dynamics is an RTS dynamics $(S, \Phi)$ such that its state space $S$ carries a vector lattice 
structure which is subject to the following compatibility axioms: (i) $\Phi\left(t, s+s^{\prime}\right)=\Phi(t, s)+\Phi\left(t, s^{\prime}\right)$; (ii) $\Phi(t, \lambda s)=$ $\lambda . \Phi(t, s)$; (iii) $s \leq s^{\prime} \Rightarrow \Phi(t, s) \leq \Phi\left(t, s^{\prime}\right), \forall t \geq 0$.

Remark 1: (i) Any latticeal dynamics is topological. (ii) Any analytical or Hilbertean dynamic is also latticeal.

The life time of the system $(S, \Phi)$ is the map $\zeta: S \rightarrow$ $[0, \infty]$ defined by $\zeta(s)=\inf \{t \geq 0 \mid \Phi(t, s)=\Delta\}$. We can suppose without loosing the generality that for all $s \in S$ the life time $\zeta(s)>0$. For each $s \in S$ the abstract trajectory starting from $s$ is $\Gamma_{s}=\{\Phi(t, s) \mid t \in[0, \zeta(s))\}$. A symbolic dynamics $(S, \Phi)$ with deadlock is called transient if there exists a sequence of measurable $\left(A_{n}\right)_{n \in \mathbb{N}}$ such that and $m\left\{t \in[0, \infty) \mid \Phi(t, s) \in A_{n}\right\}<\infty ; \forall s \in S$, where $m$ is the Lebesgue measure.

Let $S$ be the set of positive elements of a vector lattice. An element $u \in S$ is called cancellable if $s+u \leq q+u \Rightarrow s \leq q$ and archimedian if $\inf _{n} \frac{1}{n} u=0$. Let us denote by $s_{n} \nearrow s$ the situation when $s_{1} \leq s_{2} \leq \ldots \leq s_{n} \Rightarrow \exists \vee_{n} s_{n} \in S$. A map $f: S \rightarrow S$ is called increasingly continuous when $s_{n} \nearrow s \Rightarrow f\left(s_{n}\right) \nearrow f(s)$.

In the practice of mathematical modelling with differential equations or stochastic processes, the elements of interest (solutions, flows, classes of trajectories) are embedded in a larger functional space. This is because, in most cases, these elements form mathematical structures poor in useful properties. Within a larger structure, the mathematical properties are then used to characterize classes of interesting elements. In technical terms, these classes constitute the image of special operators that map the state space into itself. Here, we consider a quite general class of such operators that we call sweepings.

Definition 4: A map $V: S \rightarrow S$ is called a sweeping if it is additive, monotone increasing and increasingly continuous.

Example 2: For every latticeal dynamics, the map $s \rightarrow$ $\Phi(t, s)$ is a sweeping on $S$.

Example 3: Let $(X, \mathcal{B})$ a measurable space, and $\mathcal{M}$ the cone of all positive measures on $(X, \mathcal{B})$. Suppose that $\mu$ is a fixed measure on $(X, \mathcal{B})$. Then $V_{\mu}: \mathcal{M} \rightarrow \mathcal{M}$ given by $V_{\mu}(\varsigma):=\vee_{n}(\varsigma \wedge n \mu)$ is a sweeping on $\mathcal{M}$. Moreover, the following representation holds $V_{\mu}(\varsigma)=\vee\left\{\mu^{\prime} \mid \mu^{\prime} \ll \mu\right\}$, where the notation $\mu^{\prime} \ll \mu$ means that $\mu^{\prime}$ is absolutely continuous w.r.t. $\mu$.

For any RTS dynamics $(S, \Phi)$ let us consider the set $S^{+}$ of all valuations $S \mapsto[0,+\infty]$.

Definition 5: A latticeal valuation is a map $\mu \in S^{+}$which have the following properties: additivity: $\mu\left(s+s^{\prime}\right)=\mu(s)+$ $\mu\left(s^{\prime}\right)$; homogeneity: $\mu(a . s)=a . \mu(s), \forall a \geq 0$; monotonicity: $s \leq s^{\prime} \Rightarrow \mu(s) \leq \mu\left(s^{\prime}\right)$; continuity from below: $s_{n} \nearrow$ $s \Rightarrow \mu\left(s_{n}\right) \nearrow \mu(s)$.

Let $S^{*}$ be the set of all latticeal valuations $\mu$ from $S^{+}$. When organized with pointwise addition, scalar multiplication and order $S^{*}$ becomes isomorphic with the set of positive elements of a vector lattice, in which even $\vee_{i} \mu_{i}$ exists for increasing families $\left(\mu_{i}\right)_{i} . S^{*}$ is called the dual of $S$, in a sense that will be defined below. On $S^{*}$, we may define the canonical extension of $\Phi$, denoted by $\Phi^{*}$ as $\Phi^{*}: S^{*} \rightarrow S^{*}$, given by $\Phi^{*} \mu(s)=\mu(\Phi s)$ for all $s \in S$, $\mu \in S^{*}$. Each sweeping $V: S \rightarrow S$ has an adjoint sweeping, defined as $V^{*}: S^{*} \rightarrow S^{*}$ by $V^{*}(\mu)(s):=\mu(V s)$, for all $\mu \in S^{*}$ and $s \in S$.

Lyapunov elements: In the theory of stochastic processes, the excessive functions are called sometimes Lyapunov functions. Symbolically, we may define the supermedian and symbolic Lyapunov elements corresponding to a latticeal dynamics $(S, \Phi)$ as follows:

- an element $s \in S$ is called supermedian w.r.t. $\Phi$ if $\Phi(t, s) \leq$ $s$, for all $t \geq 0$. The cone of supermedian elements is denoted by $S_{\text {sup }}$.

- A supermedian element $s \in S_{\text {sup }}$ is called symbolic Lyapunov function if $\vee_{t>0} \Phi(t, s)=s$. The cone of symbolic Lyapunov functions is denoted by $S_{e}$.

Definition 6: A family $\mathcal{V}=\left(V_{\alpha}\right)_{\alpha>0}$ of sweepings on $S$ is called resolvent if the following two axioms are satisfied: (a) $V_{\alpha} \circ V_{\beta}=V_{\beta} \circ V_{\alpha}, \forall \alpha, \beta>0$ ( commutativity); (b) $V_{\alpha}=V_{\beta}+(\beta-\alpha) V_{\alpha} \circ V_{\beta}, \forall 0<\alpha<\beta$ (resolvent equation).

The supermedian and symbolic Lyapunov functions can defined w.r.t. to the resolvent $\mathcal{V}=\left(V_{\alpha}\right)_{\alpha>0}$ associated to the symbolic dynamics. This resolvent is defined as the following Laplace transform: $V_{\alpha} s=\int_{0}^{\infty} e^{-\alpha t} \Phi(t, s) d t$. Note that if $\Phi$ is defined on $S$ then $V_{\alpha}$ is defined on $S^{*}$ and viceversa.

Simulation morphisms: Suppose that $(S, \Phi)$ and $(Q, \Psi)$ are two latticeal dynamics. We denote by $S_{e}$ and $Q_{e}$ their cones of symbolic Lyapunov functions.

Definition 7: We call a morphism any map $\varphi: Q \rightarrow S$ such that the following axioms hold: (i) additivity: $\varphi(q+$ $\left.q^{\prime}\right)=\varphi(q)+\varphi\left(q^{\prime}\right)$, for all $q, q^{\prime} \in Q$; (ii) monotonicty: $q \leq$ $q^{\prime} \Rightarrow \varphi(q) \leq \varphi\left(q^{\prime}\right)$; (iii) continuity on increasing sequences: $q_{i} \nearrow q \Rightarrow \varphi\left(q_{i}\right) \nearrow \varphi(q)$.

Any morphism $\varphi$ has an adjoint, denoted $\varphi^{*}: S^{*} \rightarrow Q^{*}$ defined by $\varphi^{*}(\mu):=(q)=\mu(\varphi(q))$.

Definition 8: A morphism $\varphi: Q \rightarrow S$ is called a simulation morphism between the latticeal dynamics $(Q, \Psi)$ and $(S, \Phi)$ if any trajectory on $Q$ is transformed via $\varphi$ (is simulated) into a trajectory on $S$, i.e. $\varphi[\Psi(t, q)]=\Phi[t, \varphi(q)]$, for all $t \geq 0, q \in Q$.

Proposition 1: If $\varphi: Q \rightarrow S$ is a simulation morphism then its adjoint $\varphi^{*}: S^{*} \rightarrow Q^{*}$ is also a simulation morphism.

Proof: $\Psi^{*}\left(t, \varphi^{*} \mu\right)(q)=\left(\varphi^{*} \mu\right)[\Psi(t, q)]=$ $\mu[\varphi[\Psi(t, q)]]=$

$=\mu(\Phi[t, \varphi(q)])=\Phi^{*}(t, \mu)(\varphi(q))=\varphi^{*}\left[\Phi^{*}(t, \mu)\right](q)$.

Proposition 2: The symbolic Lyapunov functions are preserved through a simulation morphism, i.e. $q \in Q_{e} \Rightarrow$ $\varphi(q) \in S_{e} ; \mu \in\left(S^{*}\right)_{e} \Rightarrow \varphi^{*}(\mu) \in Q^{*}$.

The proof is trivial, and it is based on the definitions of the Lyapunov functions and simulation morphism.

For $(S, \Phi)$, let us denote $\varphi_{t}(s):=\Phi(t, s)$, for all $s \in S$. The symbolic dynamics structure provides in a canonical way a family of simulation morphisms $\left(\varphi_{t}\right)_{t>0}$ as follows. For all $t>0, \varphi_{t}: S \rightarrow S$ is a simulation morphism. Indeed, $\Phi\left(t^{\prime}, \varphi_{t}(s)\right)=\Phi\left(t^{\prime}, \Phi(t, s)\right)=\Phi\left(t+t^{\prime}, s\right)=$ $\Phi\left(t, \Phi\left(t^{\prime}, s\right)\right)=\varphi_{t}\left(\Phi\left(t^{\prime}, s\right)\right), \forall t^{\prime}>0$. 
If $(Q, \Psi)$ is a symbolic dynamics and $S$ is only a set, and $\varphi: Q \rightarrow S$ is a surjective map, then $S$ may be equipped with a structure of a symbolic dynamics as follows. To have well defined operations on $S$, we need the following further assumptions: (A1) If $\varphi(q)=\varphi\left(q^{\prime}\right)$ and $\varphi(p)=\varphi\left(p^{\prime}\right)$ then $\varphi(q+p)=\varphi\left(q^{\prime}+p^{\prime}\right)$. (A2) If $\varphi(q)=\varphi\left(q^{\prime}\right)$ and $\alpha \geq 0$ then $\varphi(\alpha q)=\varphi\left(\alpha q^{\prime}\right)$. In this way, the transfer of the algebraic operations from $Q$ to $S$ is well defined. Furthermore, to define the order relation on $S$, we need to impose another assumption: (A3) If $q \leq q^{\prime} \leq q^{\prime \prime}$ and $\varphi(q)=\varphi\left(q^{\prime \prime}\right)$ then $\varphi(q)=\varphi\left(q^{\prime}\right)$. With these assumptions in hand, we may define the order relation as follows: $s \leq s^{\prime}$ if $\forall q \in Q$ with $\varphi(q)=s, \exists q^{\prime} \in Q$ such that $\varphi\left(q^{\prime}\right)=s^{\prime}$, and $q \leq q^{\prime}$.

A simulation morphism can be used to construct in a natural way an equivalence relation. Let $\varphi: Q \rightarrow S$ be a simulation morphism. Let $\sim$ be the equivalence relation on $S$ w.r.t. $\varphi$, i.e. $q^{\sim} q^{\prime} \Longleftrightarrow \varphi(q)=\varphi\left(q^{\prime}\right)$, and $Q / \sim$ the quotient latticeal w.r.t. . The space $Q / \sim$ can be endowed with an algebraic structure using the previous method and the algebraic structure of $Q$. Moreover, one can construct another simulation morphism from $Q / \sim$ to $\varphi(Q)$. All these ideas are summarized in the following theorem.

Theorem 3: (I) $Q / \sim$ can be canonically equipped as a latticeal dynamics, and the projection $\pi: Q \rightarrow Q / \sim$ is a simulation morphism.

(II) $\varphi(Q)$ has a canonical structure as a latticeal dynamics with the inclusion $i: \varphi(Q) \hookrightarrow S$ as a simulation morphism. There exists a canonical decomposition $Q \stackrel{\pi}{\rightarrow} Q / \sim \stackrel{\bar{\varphi}}{\longrightarrow}$ $\varphi(Q) \stackrel{i}{\hookrightarrow} S$, where $\bar{\varphi}$ is bijective.

\section{SyMbOLIC DYNAMICS FOR STOCHASTIC REAL TIME SYSTEMS}

In order to model uniformly the Markov processes and deterministic dynamical systems within the framework of symbolic dynamics, we need to abstract away some of their common properties. This unifying method derives from the so-called weak solutions of differential equations. For equations where solutions can not be computed, the existence and important analytical properties of the solutions can be established. The key point is to consider a larger space of elements that contains the solutions. A typical example of such a space constitutes $\mathbf{B}(X)$. The differential operator becomes then a linear operator on a subset of this large space. For a Markov process, this operator is nothing else but its infinitesimal generator. Reasoning about this operator is usually done via a time-indexed family of "approximating" simpler operators, represented by the operators of the Markovian semigroup.

Recall that a family $\left\{P_{t}: \mathbf{B}(X) \rightarrow \mathbf{B}(X), t \geq 0\right\}$ of linear operators on $\mathbf{B}(X)$ is called semigroup of operators if the following conditions are satisfied: (i) semigroup property: $P_{t} P_{s}=P_{t+s}, t, s \geq 0$; (ii) contraction property: $\left\|P_{t} f\right\| \leq$ $\|f\|, f \in \mathbf{B}(X)$.

In addition, if $\lim _{t \rightarrow 0} P_{t} f=f$, then $\left(P_{t}\right)$ is called strongly continuous semigroup.If $\left(P_{t}\right)$ is a strongly continuous contraction semigroup then $D(\mathcal{L})$ is dense. In addition, $\mathcal{L}$ is closed, i.e. if $f_{n} \in D(\mathcal{L})$ converges to $f$ and $\mathcal{L} f_{n}$ converges to $g$ then $g \in D(\mathcal{L})$ and $\mathcal{L} f=g$.

The Markovian semigroup associated with a Markov process is a semigroup of operators, due to the ChapmanKolmogorov equation [6].

If $(X, \phi)$ is a deterministic dynamical system one can associate the semigroup of operators $\mathcal{P}=\left(P_{t}\right)_{t>0}$ defined by

$$
P_{t} f(x)=f(\phi(t, x))
$$

for all functions $f \in \mathbf{B}(X)$. For each $t>0$, the function $P_{t} f$ applied in a state $x \in X$ is the image of the measurable function $f$ at that point corresponding to the time $t$ of the flow $\phi(\cdot, x)$ (which starts in $x$ at time 0 ). In other words, $P_{t} f$ describes the abstract state of the system at time $t$ or how a logical formula $f$ is changed after the time $t$. If, in the semigroup formula, we take $f=I_{A}$ with $A \in \mathcal{B}$ (the indicator function of a measurable set $A$ ) then $P_{t} I_{A}(x)=$ $I_{A}(\phi(t, x))$, i.e. it takes the value one if and only if $\phi(t, x) \in$ $A$, otherwise it is equal to zero (see [12] and the references therein, for more properties of the semigroup associated to a deterministic dynamical system).

Remark 2: The semigroup formula (3) can be derived as a particular case of Markov semigroup, taking the transition probabilities $p_{t}(x, \cdot)=\delta_{\phi(t, x)}(\cdot), t \geq 0$, where $\delta_{\phi(t, x)}$ is the Dirac distribution corresponding to $\phi(t, x)$.

The operator resolvent can be defined using the same formula (2) like for Markov processes.

Remark 3: For a deterministic dynamical system $\phi$, if $A \in \mathcal{B}$ then $V I_{A}(x)$ is exact the Lebesgue measure of those moments of time $t \geq 0$ for which the trajectory $\Gamma_{x}$ has a non-empty intersection with $A$.

The following definition is inspired by a condition from the Hille-Yosida theorem (Th. 2.6, Chapter 1 in [6]).

Definition 9: A linear operator $\mathcal{L}$ has the Hille-Yosida property if for all $\lambda>0$, the operator $\lambda I-\mathcal{L}$ has an everywhere defined inverse $R(\lambda, \mathcal{L})$ such that $\|\lambda R(\lambda, \mathcal{L})\| \leq$ 1. To say $\lambda I-\mathcal{L}$ has an everywhere defined inverse means that the operator $\lambda I-\mathcal{L}$ is injective on the domain of $\mathcal{L}$ and that its range is all of $X$.

The Hille-Yosida theorem gives necessary and sufficient conditions for a linear operator to be the generator of a strongly continuous, positive contraction semigroup. To the semigroup $\mathcal{P}$ one can associate its operator resolvent $\mathcal{V}$ and its infinitesimal generator $\mathcal{L}$. Conversely, given an operator semigroup $\mathcal{P}$, one can check if it might be associated to a Markov process (for necessary and sufficient conditions to ensure that the semigroup can be interpreted as a semigroup of conditional expectations see Th. 2.2, Chapter 4, [6]).

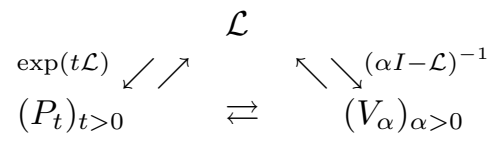

In the conclusion of this subsection, we have to point out that properties of the operator semigroup for a Markov process, are well understood (Dynkin formula, forward/backward Kolmogorov equations, etc.). Therefore, the 
idea to base an abstraction approach on the properties of this semigroup arises naturally.For dynamical systems, governed

by ordinary differential equations the infinitesimal generator is the Lie derivative. If this is not available, usually we are reasoning about their properties in terms of the operator semigroup.

With all the ingredients prepared in the previous subsection, we are ready now to introduce an unifying concept for deterministic dynamical systems and Markov processes.

Suppose that we have given a Markov process $\mathbf{M}$ or a dynamical system $\phi$ (that is a degenerate Markov process). Mainly the Markov dynamics is described by the tuple $\Im=$ $(X, \mathcal{P})$ defined as follows: (i) $X$ is the state space (with a topological structure Polish/analytic/Lusin space) of the underlying system; (ii) $\mathcal{P}=\left(P_{t}\right)$ is the associated Markov operator semigroup on $\mathbf{B}(X)$.

We can define a preorder relation $\prec$ on $X$ as

$$
x \prec y \Longleftrightarrow V f(y) \leq V f(x), \forall f \in \mathbf{B}(X), f \geq 0 .
$$

where $V$ is the kernel operator.

Using (4), we can define a preorder relation $\prec_{M}$ associated to $\mathbf{M}$. Intuitively, $\prec_{\mathbf{M}}$ is the order on the trajectories of M. In particular, if $\mathbf{M}$ degenerates in a semi-dynamical system, $\prec_{M}$ is exactly the order relation on the trajectories. We denote $x \prec_{\phi} y$ if there exists $t \in[0, \infty)$ such that $y=\phi(t, x)$. If the system under consideration is transient then $\prec_{\phi}$ is an order relation [12]. This order relation can be characterized using the initial resolvent kernel via (4).

Let us consider $(X, \mathcal{P})$ as a representation for a Markov process $\mathbf{M}$, or a dynamical systems $\phi$. We extend $\mathcal{P}$ with $P_{0}=I$, where $I$ is the identity operator.

Let us define the following Markovian symbolic dynamics $\Im=(S, \Phi)$ : (i) The "state space" is $S:=\mathbf{B}(X)$, where $\mathbf{B}(X)$ is the lattice of the bounded positive measurable functions on $X$; (ii) The "dynamics" is given by $\Phi(t, f):=$ $P_{t} f$; for all $f \in \mathbf{B}(X)$.

The properties of the symbolic dynamics can be deduced from the Markovian properties of $\mathcal{P}$ and from the definition of this semigroup as an integral w.r.t. a probability measure. Note that the state space of this symbolic dynamics has a richer algebraic/topological structure compared with the structure of the state space of the given process.

\section{Symbolic Bisimulation}

In this section we define a categorical concept of bisimulation for symbolic dynamics. The main goal is to show that in the category of symbolic dynamics obtained using simulation morphisms as arrows, we can obtain a bisimulation relation that is defined via a category theory methodology.

A Category of symbolic dynamics: We define the category SD of symbolic dynamics, which has as: (a) $o b$ jects - Markovian symbolic dynamics; (b) arrows - zigzag morphisms, which will be defined below. The aim of this subsection is to give an appropriate definition of the zigzag morphisms (and of simulation morphisms) between such dynamics, which will allow us to define a general concept of unifying bisimulation in this category.

Let $\Im^{1}$ and $\Im^{2}$ be two objects of SD. The state space of $\Im^{1}$ (resp. $\Im^{2}$ ) is $\mathbf{B}\left(X^{(1)}\right)$ (resp. $\mathbf{B}\left(X^{(2)}\right)$ ). Let $\varphi$ : $\mathbf{B}\left(X^{(1)}\right) \rightarrow \mathbf{B}\left(X^{(2)}\right)$ be a morphism of symbolic dynamics.

Definition 10: A surjective simulation morphism $\varphi$ : $\mathbf{B}\left(X^{(1)}\right) \rightarrow \mathbf{B}\left(X^{(2)}\right)$ between the dynamics $\Im^{2}$ and $\Im^{1}$ is called zigzag morphism, if for almost all $t \geq 0$ (i.e. except with a zero Lebesgue measure set of times) the following equality holds

$$
P_{t}^{2} \circ \varphi=\varphi \circ P_{t}^{1}
$$

where $\left(P_{t}^{1}\right)$ (resp. $\left(P_{t}^{2}\right)$ ) is the semigroup of operators associated to $\Im^{1}$ (resp. $\Im^{2}$ ).

The relation (5) is known in the literature as the Dynkin intertwining relation [9]. This definition illustrates that the simulating dynamics can make all the transitions of the simulated one with the same transition probabilities than in the dynamics being simulated. Moreover, this illustrates that the zigzag morphism introduced in this section is a natural generalization of the similar concept defined for particular classes of Markov processes in [8].

Usually, such a morphism $\varphi$ is obtained from a surjective measurable map $\psi: X^{(2)} \rightarrow X^{(1)}$ through the relation

$$
\varphi(f):=f \circ \psi ; \forall f \in \mathbf{B}\left(X^{(1)}\right)
$$

Roughly speaking, this means that whilst the process corresponding to $\Im^{2}$ evolves from $u$ to $\psi^{-1}(A)$ ( $A \in$ $\mathcal{B}\left(X^{(1)}\right)$ ) on a trajectory with a given probability, the process corresponding to $\Im^{1}$ evolves from $\psi(u)$ to $A$ with the same probability.

The following result is very important in the context of simulation and bisimulation problems. It gives a methodology to "lift" the simulation from the state space level to the level of Lyapunov functions defined on the state space.

Proposition 4: If a zigzag morphism $\varphi:: \mathbf{B}\left(X^{(1)}\right) \rightarrow$ $\mathbf{B}\left(X^{(2)}\right)$ can be represented as in (6) then it induces a simulation morphism between the cones of Lyapunov functions:

$$
\widehat{\varphi}: \mathcal{E}_{1} \rightarrow \mathcal{E}_{2} ; \widehat{\varphi}(s):=s \circ \psi
$$

This result shows that for the purpose of simulation we may define the simulation morphism only on the Lyapunov function cones. This might be an interesting approach when representations of such cones are available.

Bisimulation Properties: We consider the category SD defined in the previous section. Then, we define the bisimulation between two processes in this category as the existence of a sink of zigzag morphisms between them.

Let $\Im^{1}$ and $\Im^{2}$ be two objects in SD. $\Im^{1}$ is bisimilar to $\Im^{2}$ (written $\Im^{1} \sim \Im^{2}$ ) if there exists a sink of zigzag morphisms between them, i.e. there exists $\Im^{12}$ (object in SD) and two zigzag morphisms $\varphi^{1}$ and $\varphi^{2}$ as follows: $\mathbf{B}\left(X^{(1)} \stackrel{\varphi^{1}}{\longrightarrow} \mathbf{B}\left(X^{12}\right) \stackrel{\varphi^{2}}{\longleftarrow} \mathbf{B}\left(X^{(2)}\right)\right.$.

Theorem 5: The category SD has semi-pushout.

Proof: Suppose that $\Im^{1}, \Im^{2}, \Im$ are three dynamics defined on $\mathbf{B}\left(X^{(1)}\right), \mathbf{B}\left(X^{(2)}\right), \mathbf{B}(X)$, respectively. Assume 
that there exist two zigzag morphisms

$$
\mathbf{B}\left(X^{(2)}\right) \stackrel{\varphi^{2}}{\longleftarrow} \mathbf{B}(X) \stackrel{\varphi^{1}}{\longrightarrow} \mathbf{B}\left(X^{(1)}\right)
$$

Our goal is to prove that there exists another object $\Im^{0} \in \mathbf{S D}$ and two zigzag morphisms $j^{1}: \mathbf{B}\left(X^{(1)}\right) \rightarrow \mathbf{B}\left(X^{(0)}\right)$ and $j^{2}: \mathbf{B}\left(X^{(2)}\right) \rightarrow \mathbf{B}\left(X^{(0)}\right)$ such that the following diagram commutes:

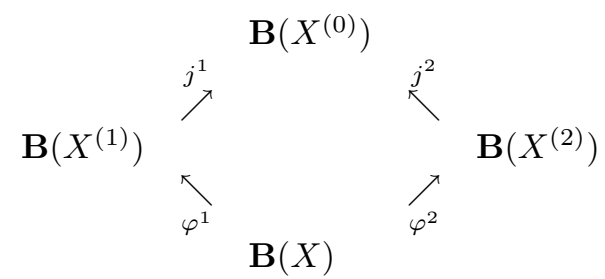

First step is to construct the desired dynamics $\Im^{0}$. The state space of $\Im^{0}$ will be defined as follows: $\widehat{\mathbf{B}}\left(X^{(0)}\right):=$ $\left\{\left(f^{1}, f^{2}\right) \in \mathbf{B}\left(X^{(1)}\right) \times \mathbf{B}\left(X^{(2)}\right) \mid f^{1}=\varphi^{1}(f), f^{2}=\varphi^{2}(f)\right\}$. Let now have a closer look at the space $\widehat{\mathbf{B}}\left(X^{(0)}\right)$ : (a) Since $\varphi^{1}$ and $\varphi^{2}$ are supposed surjective, we have: $\widehat{\mathbf{B}}\left(X^{(0)}\right) \subset$ $\mathbf{B}\left(X^{(1)}\right) \times \mathbf{B}\left(X^{(2)}\right)$; (b) It is a norm space because it can be naturally equipped with the norm of $\mathbf{B}\left(X^{(1)} \times X^{(2)}\right)$.

Now we can define the semigroup of $\Im^{0}$ as the restriction to $\widehat{\mathbf{B}}\left(X^{(0)}\right)$ of the semigroup corresponding to $\Im^{1} \otimes \Im^{2}$ (the product of $\Im^{1}, \Im^{2}$ ). Denote by $\mathcal{L}^{1}, \mathcal{L}^{2}$ the generators associated with $\left(P_{t}^{1}\right)$ and $\left(P_{t}^{2}\right)$ (the semigroups of $\Im^{1}$ and $\left.\Im^{2}\right)$. According to [16], the product process $\Im^{1} \otimes \Im^{2}$ has the generator given by the smallest closed extension of the operator defined on $D\left(\mathcal{L}^{1}\right) \otimes D\left(\mathcal{L}^{2}\right)$ by Trotter formula [16]: $\mathcal{L}(f \otimes g)=\mathcal{L}^{1}(f) \otimes g+f \otimes \mathcal{L}^{2}(g)$, where $f \in D\left(\mathcal{L}^{1}\right)$ and $g \in D\left(\mathcal{L}^{2}\right)$. The domain of the generator $\mathcal{L}$ denoted by $D(\mathcal{L})$ includes $D\left(\mathcal{L}^{1}\right) \otimes D\left(\mathcal{L}^{2}\right)$. The generator $\mathcal{L}^{0}$ of $\Im^{0}$ coincides with the generator $\mathcal{L}$ of $\Im^{1} \otimes \Im^{2}$ for all functions $f \in \widehat{\mathbf{B}}\left(X^{(0)}\right)$.The second step is to find suitable zigzag morphisms $j^{1}$ and $j^{2}$. These can be taken as the inclusion maps. The surjectivity of $j^{1}$ or $j^{2}$ can be easily derived using the surjectivity of $\varphi^{1}$ and $\varphi^{2}$ and the definition of $\widehat{\mathbf{B}}\left(X^{(0)}\right)$. Using Trotter formula, it follows that these projection maps are indeed zigzag morphisms.

An immediate corollary of the existence of semi-pullbacks in the category SD is the following.

Proposition 6: The bisimulation in the category SD is an equivalence relation.

Example 4: The following systems are bisimilar: (a) $x^{\prime}=$ $x^{2}+1$ and $y^{\prime}=y^{2}+2 y+2$ via the relation $x=y+1$; (b) $x^{\prime}=2 x+1$ and $\left\{\begin{array}{l}u^{\prime}=2 u \\ v^{\prime}=2 v-\frac{1}{2}\end{array} \quad\right.$ via the relation $x=u-2 v$.

\section{Conclusions}

In this paper, we have developed a mathematical setting to model new concepts from hybrid systems into general systems theory [13].

The advantages of this approach are as follows: (a) the elegance of the mathematical setting that combines algebra and functional analysis; (b) both discrete and continuous behaviors are instances of the same concept; (c) unification and uniformity in the study the essential properties of deterministic and stochastic behaviors; (d) the framework shows that the mathematical models play the role of formal specifications (for example, the bisimulation and similarity relations show that mathematical models defined in different mathematical theories describe the same dynamical evolutions).

This framework can be used in many ways. The specification languages developed for discrete dynamical systems can be easily extended to continuous time behaviors by a simple embedding of the standard denotational semantics into the framework. Concepts like simulation, refinement, formal verification that are well understood and developed for discrete time systems can be extended in a straightforward manner to continuous time systems. The common axiomatization of deterministic and stochastic processes shows that the stochastic approach provides a system theoretic abstraction of more complex deterministic behaviors.. In this context, it might be interesting to investigate computability questions.

The theory of symbolic simulations will be developed towards a full systems theory framework by considering topics like stability, invariance principles, controllability, refinement, limit systems, interconnections and hierarchical structures, and more issues related to verification.

\section{REFERENCES}

[1] Aaron, D. A.: A Categorical Theory of Hybrid Systems. PhD dissertation, University of California, Berkeley (2006).

[2] Bensoussan, A.: "Stochastic Control by Functional Analysis Methods". Elsevier (1982).

[3] Bujorianu, M.L., Lygeros, J., Langerak, R.: Reachability Analysis of Stochastic Hybrid Systems by Optimal Control. Springer LNCS 4981 (2008): 610-613.

[4] Bujorianu, M.L., Bujorianu, M.C.: A Theory of Symbolic Dynamics for Hybrid Systems. Submitted.

[5] Davis, M.H.A.: "Markov Models and Optimization" Chapman \& Hall, (1993).

[6] Ethier, S.N., Kurtz, T.G.: "Markov Processes: Characterization and Convergence”. New York: John Wiley and Sons, (1986).

[7] Bujorianu, M. L. ; Lygeros, J.: Toward a general theory of stochastic hybrid systems. Stochastic hybrid systems, LNCIS 337(2006): 3-30.

[8] Bujorianu, M.L., Lygeros, J., Bujorianu, M.C.: Bisimulation for General Stochastic Hybrid Systems. In [14]: 198-214.

[9] Dynkin, E.B.: "Markov Processes". Vol.1. Berlin Heidelberg New York: Springer (1965).

[10] Haghverdi, E., Tabuada, P., Pappas, G.J.: Open maps, alternating simulations and control synthesiss. Springer LNCS 3170, (2004): 466480.

[11] Hinrichsen, D., Pritchard, A.J.: "Mathematical Systems Theory I: Modelling State Space Analysis, Stability and Robustness". Springer (2000).

[12] Hmissi, M.: Semi-groupes Deterministes. Sem. Th. Potentiel 9, Paris, LNM 1393, (1989): 135-144.

[13] Lin, Y.: "General Systems Theory: A Mathematical Approach". Kluwer Academic, (1999).

[14] Morari, M., Thiele, L. (Eds.), Proc. of Hybrid Systems: Computation and Control, 8th International Workshop, LNCS 3414 (2005).

[15] Saperstone, S. H.: "Semidynamical Systems in Infinite Dimensional Spaces ". Springer Verlag (1981).

[16] Trotter, H.F.: On the Product of Semigroups of Operators. Proc. Amer. Math. Soc. 10 (1959): 545-551.

[17] Temam, R.: "Infinite-Dimensional Dynamical Systems in Mechanics and Physics". Springer (1997). 